Research Paper

\title{
PEGylated Phospholipid Micelle-Encapsulated Near-Infirared PbS Quantum Dots for in vitro and in vivo Bioimaging
}

\author{
Rui Hư, 2 , Wing-Cheung Law², Guimiao Lin¹, Ling Ye³, Jianwei Liu³, Jing Liu³, Jessica L. Reynolds ${ }^{4}$ and \\ Ken-Tye Yong ${ }^{1 凶}$
}

1. School of Electrical and Electronic Engineering, Nanyang Technological University, Singapore 639798, Singapore.

2. Institute for Lasers, Photonics and Biophotonics, University at Buffalo, State University of New York, Buffalo, NY 14260-4200, USA.

3. Institute of Gerontology and Geriatrics, Chinese PLA General Hospital, Beijing 100853, China.

4. Division of Allergy, Immunology and Rheumatology, Department of Medicine, State University of New York at Buffalo, Innovation Center, 640 Ellicott Street, Buffalo, NY 14203.

Corresponding author: Ken-Tye Yong, PhD, School of Electrical and Electronic Engineering, Nanyang Technological University, Singapore 639798, Singapore Tel: +65-6790-5444, email: ktyong@ntu.edu.sg.

(C) Ivyspring International Publisher. This is an open-access article distributed under the terms of the Creative Commons License (http://creativecommons.org/ licenses/by-nc-nd/3.0/). Reproduction is permitted for personal, noncommercial use, provided that the article is in whole, unmodified, and properly cited.

Received: 2012.02.24; Accepted: 2012.06.17; Published: 2012.08.01

\begin{abstract}
Surface modification and functionalization of bioconjugated quantum dots (QDs) has drawn great attention for the past few years due to their wide applications in biomedical research. In this contribution, we demonstrate the use of PEGylated phospholipid micelles to encapsulate near infrared emitting ultra-small lead sulfide (PbS) QDs for in vitro and in vivo imaging. The cytotoxicity of the micelle-encapsulated QDs formulation was evaluated using MTS assay and histological analysis studies. We have found that upon encapsulating the QDs with phospholipid micelle, the toxicity of the PbS QDs is reduced, from which we envision that the PEGylated phospholipid micelle-encapsulated PbS QDs formulation can be used as theranostics probes for some selected applications in cell imaging and small animals study.
\end{abstract}

Key words: Phospholipid, Micelle encapsulation, quantum dots, bioimaging, PEG.

\section{Introduction}

Phospholipids are a set of naturally occurring amphiphilic molecules in organisms for constructing cell membranes. Typically, a phospholipid molecule contains a hydrophilic head and a hydrophobic tail. In aqueous solution, they can self assemble to three different structures, bi-layer sheet as in cell membrane, liposome and micelle [1-3]. A liposome has an enclosed bi-layer configuration with a hydrophobic core and a lipid bi-layer shell [4]. It is capable of encapsulating either hydrophilic or hydrophobic compounds in the core or the lipid region, and thus they are widely used for drug delivery applications [5-7]. A micelle, on the other hand, has a compact configuration, where in aqueous solution, the hydrophobic tails of the lipid molecules will aggregate together and they will form an oil-like core, while the hydrophilic head will form a shell interacting with the aqueous environment. They have been used as nanocarriers in encapsulating hydrophobic molecules for many biomedical applications [8, 9]. More recently, polyethylene glycol (PEG) grafted (also termed PEGylated) phospholipids are prepared and they are of great interests due to their high biocompatibility for clinical research [10-12]. Similarly, the oil-like molecules can 
be trapped in the core through hydrophobic interactions, leaving a hydrophilic shell modified with PEG molecules. More importantly, different functional groups, such as carboxyl, amino, and maleimide can be functionalized to the PEG molecules for further conjugation of biomolecules in targeted delivery applications.

Fluorescent imaging method has been extensively used in biomedical research [13-18]. For in vitro cell imaging, the sample is illuminated with an excitation light source and fluorescent signals are collected by using an appropriate set of filters. However, even with the use of filters set, the collected fluorescence signals still contain some portion of intrinsic fluorescent signals (e.g. autofluorescence) from the sample and these signals will sometimes impair the resolution of cell imaging study. For example, NADH, flavins and aromatic amino acid constituents of proteins are generating the autofluorescence signals during imaging study and they are located in the visible wavelength range [19]. To overcome this challenge, one can use near-infrared (NIR) imaging to evade the excitation of autofluorescence signals and thus increase the signal to noise ratio from the cells sample [20-23]. In addition, NIR imaging setup can be easily extended for in vivo imaging as well. To date, it is well known that to perform small animal imaging studies in the visible range is challenging for the reason that the limited depth penetration in tissue and the scattering from the tissue of visible light are two major obstacles to obtain high resolution and details of images. It is recommended that the optimal wavelength range for in vivo imaging is around 700 to 900 $\mathrm{nm}$, where the light has relatively higher penetration depth in tissue while the absorption and scattering of the tissue are at their minimum [24, 25].

Among the currently available contrast agents used in bioimaging, quantum dots (QDs) remain one of the widely used and popular optical probes to label different compartments of the cells [23, 26-29]. QDs are semiconductor nanocrystals that have sizes which is comparable or smaller than that of the exiton bohr radius of the semiconductor material, typically a few nanometers [30,31]. Also, QDs possess many unique optical features [29, 32, 33]. For example, the emission peak of QDs can be turned over a broad wavelength range by controlling their size or composition. They have high resistance to photobleaching and narrow emission line widths. QDs also have relatively large surface areas that can be functionalized with multiple biomoelcules for specific targeted delivery [34]. More importantly, different emission color of QDs can be simultaneously excited using a single wavelength light source and one can use this technique for multi- plex imaging. While QDs have been extensively exploited in the nanomedicine field, the toxicity issues of QDs have also been raised due to the unknown risk associated with the nanoparticle exposure to the biology. Thus, verifying the safety of QDs, especially the heavy metal containing candidates, is increasingly important. Toxicity studies of cadmium based QD can be easily found in the literature [35-37]. However, evaluation on lead based QDs is somehow being neglected. Truong et al. studied the in vivo toxicity of $\mathrm{PbS}$ with different surface functionalization using zebra fish model [38]. The results suggested that the toxicity was strongly associated with the surface ligands.

In this study, we hypothesize that the toxicity of lead based QD can be suppressed with proper surface modification. We have prepared PEGylated phospholipid micelle-encapsulated lead sulfide (PbS) QDs as NIR optical probes for in vitro and in vivo imaging. For in vitro study, folic acid conjugated micelle-encapsulated PbS QDs were prepared and used for targeted imaging of pancreatic cancer cells. Also, we have used the micelle-encapsulated QDs formulation for in vivo imaging. MTS assay and histological analysis of tissues sections from the major organs were used to evaluate the toxicity of the micelle-encapsulated PbS QDs formulation. The result suggests that low toxicity was observed for micelle-encapsulated PbS QDs formulation and they are suitable for some selected in vitro and small animals studies.

\section{Materials and Experimental Methods}

\section{Materials}

1,2-Diacyl-sn-Glycero-3-Phosphoethanolamine$\mathrm{N}-[$ Methoxy(Polyethylene glycol)] (mPEG-DSPE, Mw 5000), 1,2-distearoyl-sn-glycero-3-phosphoethanolamine-N-[folate(polyethylene glycol)-2000], ammonium salt (DSPE-PEG-Folate, Mw 3230), were purchased from Laysan Bio Inc and Avanti Polar Lipids Inc, respectively. Lead sulfide quantum dots ( $\mathrm{PbS}$ QDs) dispersed in toluene were purchased from Evident Technologies (US, molar ratio of $\mathrm{Pb} / \mathrm{S}$ is $1: 1$, nanocrystal $\mathrm{MW}$ is $1.1 \mathrm{e} 4 \mu \mathrm{g} / \mathrm{nmol}$, particle $\mathrm{MW}$, i.e. crystalline core plus estimated ligands, is 5.0e4 $\mu \mathrm{g} / \mathrm{nmol}$, provided by vendor). All solvents, including HPLC water, chloroform, toluene, hexane and ethanol were of reagent grade and purchased from Sigma-Aldrich. Phosphate buffered saline (PBS) 7.2 (1x) and tetradecylphosphonic acid (TDPA) are products of Invitrogen and Alfa Aesar, respectively. All chemicals were used as received and all solvents (hexane, toluene, chloroform and ethanol) were used 
without further purification.

\section{Preparation of phospholipid micelle encapsu- lated QDs}

The PbS QDs were originally dispersed in toluene. Before the encapsulation, toluene was removed and the dried QDs were re-dispersed in chloroform at a final concentration of $1 \mathrm{mg} / \mathrm{ml}$. For the encapsulation process, $2 \mathrm{ml}$ of the QD suspension was mixed with chloroform solutions of mPEG-DSPE and DSPE-PEG-Folate at a weight ratio of 1:4:0.5 (QDs:mPEG-DSPE:DSPE-PEG-Folate) in a round bottom flask. The chloroform was then evaporated using a rotary evaporator under vacuum in the water bath at room temperature. After the evaporation, $10 \mathrm{ml}$ of HPLC water was added and the flask was gently stirred for $20 \mathrm{~min}$. The resulting mixture was filtered through a $0.2 \mu \mathrm{m}$ syringe filter, followed by centrifugation at 13,000 rpm for $15 \mathrm{~min}$ and the supernatant was discarded. The precipitate was re-dispersed in PBS solution and stored in $4^{\circ} \mathrm{C}$ for future use. QDs were also encapsulated with pure mPEG-DSPE (weight ratio QDs:mPEG-DSPE = 1:4.5) following the similar procedure and the result particles were used as non-conjugated particles.

\section{Cell viability study}

Panc-1 (ATCC NO: CRL-1469) and MiaPaCa-2 (ATCC NO: CRL-1420) cells were maintained in Dulbecco's modified Eagle's medium (DMEM, Sigma) supplemented with $10 \%$ fetal bovine serum (FBS). The cells were cultured at $37^{\circ} \mathrm{C}$ in a humidified atmosphere containing $5 \% \mathrm{CO}_{2}$. For cell viability study, MTS

(3-(4,5-dimethylthiazol-2-yl)-5-(3-carboxymethoxyphe nyl)-2-(4-sulfophenyl)-2H-tetrazolium, inner salt) assay was performed $[39,40]$. Briefly, in each assay, 5000 panc-1 cells were dispensed into each well of a 96-well flat-bottom Microtiter plate and cultured overnight. Eight sets were treated with different concentrations of phospholipid micelle encapsulated QDs ranging from 0 to $250 \mu \mathrm{g} / \mathrm{ml}$ and one set treated with PBS buffer was regarded as the non-treated control. Assays were performed in triplicate and the results were averaged. The cells were subsequently incubated for $24 \mathrm{~h}$ or $48 \mathrm{~h}$ before the assay. The absorbance of formazan (produced from the cleavage of MTS by dehydrogenases in living cells) is directly proportional to the number of live cells. $10 \mu \mathrm{l}$ of MTS reagent was added to each well and thoroughly mixed after the $24 \mathrm{~h}$ or $48 \mathrm{~h}$ incubation period. One hour later, the absorbance of the mixtures at $490 \mathrm{~nm}$ was measured by a multi-well plate reader. The cell viability was obtained by normalizing the absorbance of the sample with that from the control well and expressed as percentage, assigning the viability of non-treated cells as $100 \%$.

\section{Characterization of NIR quantum dots}

The absorption spectra were obtained using a Shimadzu scanning spectrophotometer (3101PC UV-Vis-NIR) over a wavelength range from 320 to $1600 \mathrm{~nm}$. The samples were loaded into a quartz cell for measurement using the solvent as reference. The photoluminescence spectra were obtained using the Maestro small animal imaging system with red excitation. Temporal decay of the photoluminescence signal from the sample was recorded by an EasyLife $\mathrm{V}^{\mathrm{TM}}$ system (Optical Building Blocks Corporation). Samples were loaded into a $1 \mathrm{~cm}$ standard quartz cell for the measurements. A $405 \mathrm{~nm}$ laser was used as the excitation and pure solvent was used to determine the noise level. TEM images were taken by a JEOL model JEM-2010 transmission electron microscope using an acceleration voltage of $200 \mathrm{KV}$. The specimens were prepared by drop-casting the sample dispersion onto a carbon coated 300 mesh copper grid (Carbon Type-B, Ted Pella, Inc.). The excess solvent was absorbed by a filter paper underneath. The hydrodynamic size distribution of particles was obtained by a nanoparticle size analyzer (Brookhaven 90Plus, Brookhaven Instruments Corporation) using dynamic light scattering (DLS) method.

\section{Animal studies}

Four to five weeks old female nude mice and Balb/C mice were purchased from Harlan Sprague Dawley Inc. All the animal experiments and maintenance were approved by the University at Buffalo Institutional Animal Care and Use Committee (IACUC). The animal housing area was maintained at $24^{\circ} \mathrm{C}$ with a 12-hours light/dark cycle. Water and standard laboratory chow were supplied throughout the study. All animals were acclimated to the animal facility for at least 48 hours prior to experimentation. For in vivo imaging, the nude mice were administered with PEGylated phospholipid micelle encapsulated $\mathrm{PbS}$ QDs in PBS by tail vein injection. The mice were anesthetized with isoflurane at various time points post injection for imaging. For toxicity studies, the Balb/C mice were administered with PEGylated phospholipid micelle encapsulated PbS QDs through tail vein injection. 4 weeks after the injection, the $\mathrm{Balb} / \mathrm{C}$ mice were sacrificed and the tissues from the major organs were harvested and processed with standard fixation and staining procedures for histological studies. 


\section{In vitro and in vivo imaging studies}

For in vitro cell fluorescent imaging, CRi's $\mathrm{Nu}$ ance imaging system (CRI, Woburn, MA) was used. The system is equipped with a solid state liquid crystal filter for wavelength scanning with a resolution of $10 \mathrm{~nm}$ to obtain an image cube file consisting images from every scanned channel. Pancreatic cancer cells were treated with PbS QDs and incubated for 4 hours before imaging. Red filter set was applied for the cell imaging to obtain signals from the QDs. The image cube files were analyzed with the vendor software where signals from the QDs were separated by a spectral un-mixing process. A Maestro in vivo optical imaging system (CRI, Inc., US) was used for NIR fluorescent imaging of QDs injected nude mice. For acquiring the fluorescence images at different time points, the system was operated at the cube acquisition mode. The NIR filter set was applied and image cube files were taken in series while scanning the liquid crystal filter from 750nm 950nm (with $10 \mathrm{~nm}$ step). Based on the cube files, fluorescent images and the corresponding spectra were obtained by the vendor software. The fluorescent images were rendered with pseudo-colors, where the background signals were coded in green and signals from the NIR QDs in red. It should be noted that in order to get a clear vision of the whole body, the relatively low signals from the background (in green) were enhanced by the vendor software.

\section{Results and Discussion}

Figure 1 shows the steps for preparing micelle-encapsulated PbS QDs. The QDs are encapsulated in phospholipid micelles through hydrophobic interactions between the hydrophobic QDs and the diglyceride groups, i.e. the hydrophobic tails, of the phospholipid molecules. Figure 2a shows the absorption and photoluminescence profiles of the micelle-encapsulated PbS QDs formulation. The profile shows that the nanoparticles formulation can be excited over a wide range of absorption wavelength ranging from deep-red to UV range. An emission peak was observed around 900nm for the QDs formulation. Due to the limitation of the spectroscopy equipment, we were not able to measure the spectrum profile above $950 \mathrm{~nm}$. Figure $2 \mathrm{~b}$ shows the luminescence decay of the PbS QDs after excited by a pulse laser and the curve is fitted with a first order exponential function, from which the lifetime is estimated to be $877 \mathrm{~ns}$. The fluorescent lifetime of PbS QDs is consistent with previous reports. This relatively long lifetime is useful for time-gated fluorescence imaging, where the intrinsic auto-fluorescent signals from the biological samples can be removed due to the short fluorescent lifetime (typically several nanoseconds). Figure 2c and d are transmission electron microscopy (TEM) images of PbS QDs before and after the micelle encapsulation. From the TEM analysis, the average diameter of the QDs is $\sim 2 \mathrm{~nm}$ and they are highly monodispersed in size. Since the size of the PbS QDs is relatively small, several QDs are found to be encapsulated within a single micelle. As shown in the TEM picture, though several QDs are trapped within a micelle particle, the crystal structure of individual QD remains unaffected. The hydrodynamic size distribution of the micelle-encapsulated QDs was measured by dynamic light scattering (DLS) technique. The DLS data shows that the formulation has an average diameter of about $116 \mathrm{~nm}$ and no change was observed for over a period of 2 weeks.

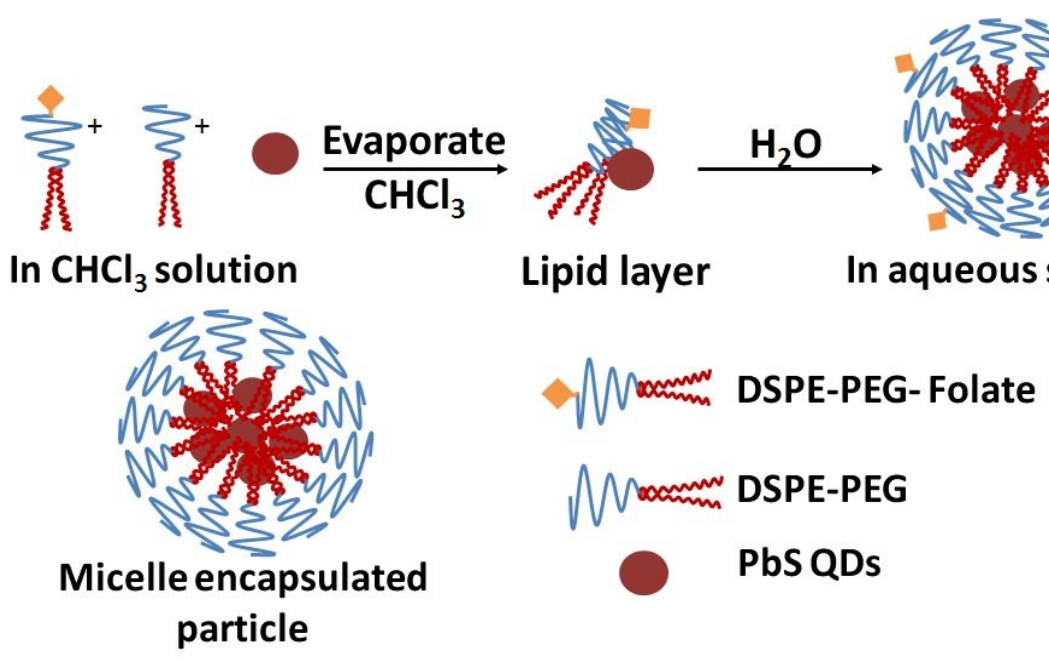

Figure I. Schematic illustration of the PEGylated phospholipid micelle encapsulation of PbS QDs. 

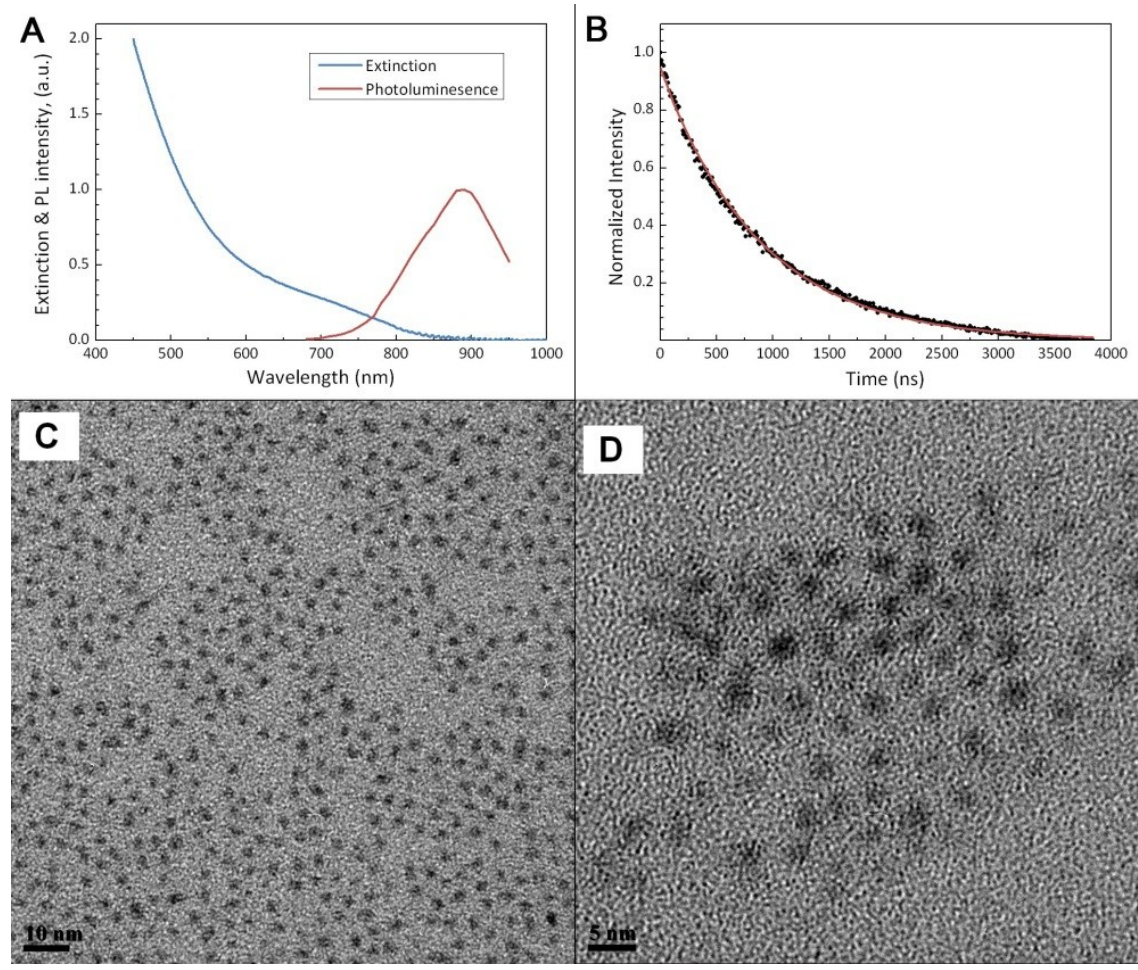

Figure 2. Characterization on the physical properties of the PbS QD nanoparticles. (a) Extinction \& photolumination (PL) profile of the QDs after micelle encapsulation. (b) Temporal decay of the photoluminescence of PbS QDs after excited by a pulse laser. Solid line shows the fitting result using a single-exponential function. (c) and (d), TEM images of the PbS QDs before and after micelle encapsulation, respectively.

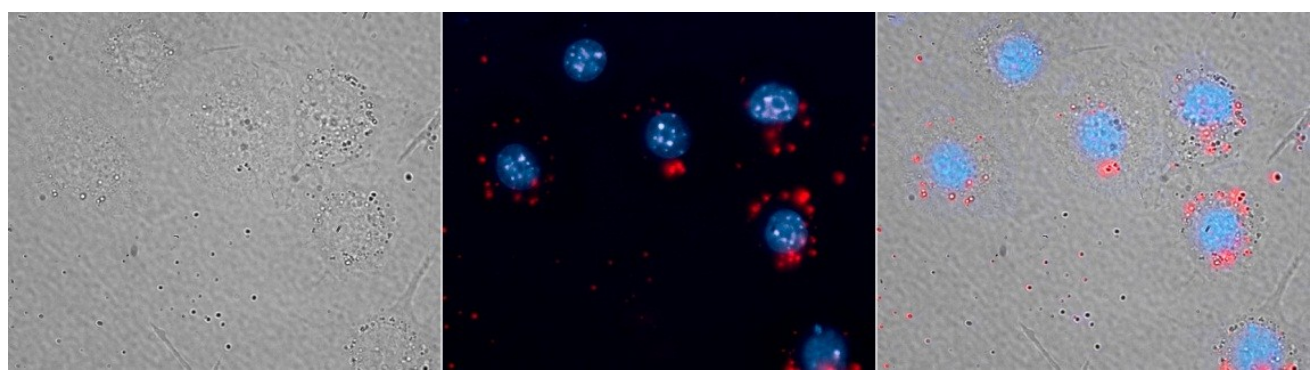

Figure 3. RAW264.7 macrophages labeled with micelle-encapsulated PbS QDs (red), the cell nucleus is stained with Hoechst 33342 (blue).

For cell imaging study, micelle-encapsulated PbS QDs were used to label RAW264.7 macrophage cells. Figure 3 shows the fluorescent image of the RAW264.7 cells treated with the QDs formulation and the cell nucleus were stained with Hoechst 3342 (pseudo-colored in blue). The signals from NIR PbS QDs are pseudo-colored in red. As shown in Figure 3, the red spots located in the cell cytoplasm clearly show the uptake of the micelle-encapsulated QDs by macrophage cells, demonstrating that the micelle-encapsulated QDs can be used as NIR optical probes for cell labeling and imaging.

In addition to labeling of macrophage cells, we have also prepared folic acid functionalized micelle-encapsulated QDs for targeted imaging of cancer cells. Folic acid is known to be very important during the period of rapid cell division and its receptors (folate receptors) are found to be overexpressed in most cancer cells [41, 42]. Human pancreatic cancer cell line, MiaPaCa-2, was used in this study. Figure 4 shows the images of MiaPaCa-2 cells treated with folic acid functionalized micelle-encapsulated NIR PbS QDs. Robust uptake of the bioconjugated micelle-encapsulated PbS QDs was observed in the MiaPaCa-2 cells. In comparison, minimal uptake was observed for unconjugated QDs. The result here demonstrates that the specificity of folic acid functionalized micelle-encapsulated QDs for targeted delivery to the MiaPaCa- 2 cancer cells.

For in vivo imaging study, nude mice were in- 
jected with PEGylated phospholipid micelle-encapsulated PbS QDs, and the treated animal is imaged at several time points. Figure 5 shows the normalized characterization spectra of the QDs injected and the background signals of a nude mouse under deep red illumination, of which the tail is labeled with the sample. The spectra are then used as references for pseudo-color rendering in the vendor software. The signals from the QDs and background are marked in red and green, respectively. It is also worth mentioning that, as seen from the spectra, the signals from the QDs are far away separated with those from the background, which will greatly increase the contrast of the image and reduce the error induced by the crosstalk between the two signal channels. Figure 6 shows the images obtained from the Maestro small animal imaging system of a mouse post-injected with QDs formulation at $1 \mathrm{~h}, 3 \mathrm{~h}, 6 \mathrm{~h}$, and $42 \mathrm{~h}$. The panel to the left shows the original unprocessed images obtained from the system. The middle panel is the signal from the QDs separated by the vendor software and the images in the right panel are pseudo-colored images showing both background and QDs signals. As shown in Figure 6, one can see that the PbS QDs are mainly accumulated in the liver and spleen after $1 \mathrm{~h}$ of injection, as denoted by the arrows. The result shows that the PEGylated phospholipid micelle encapsulated NIR PbS QDs formulation can be served as efficient high-contrast agent for small animal imaging.

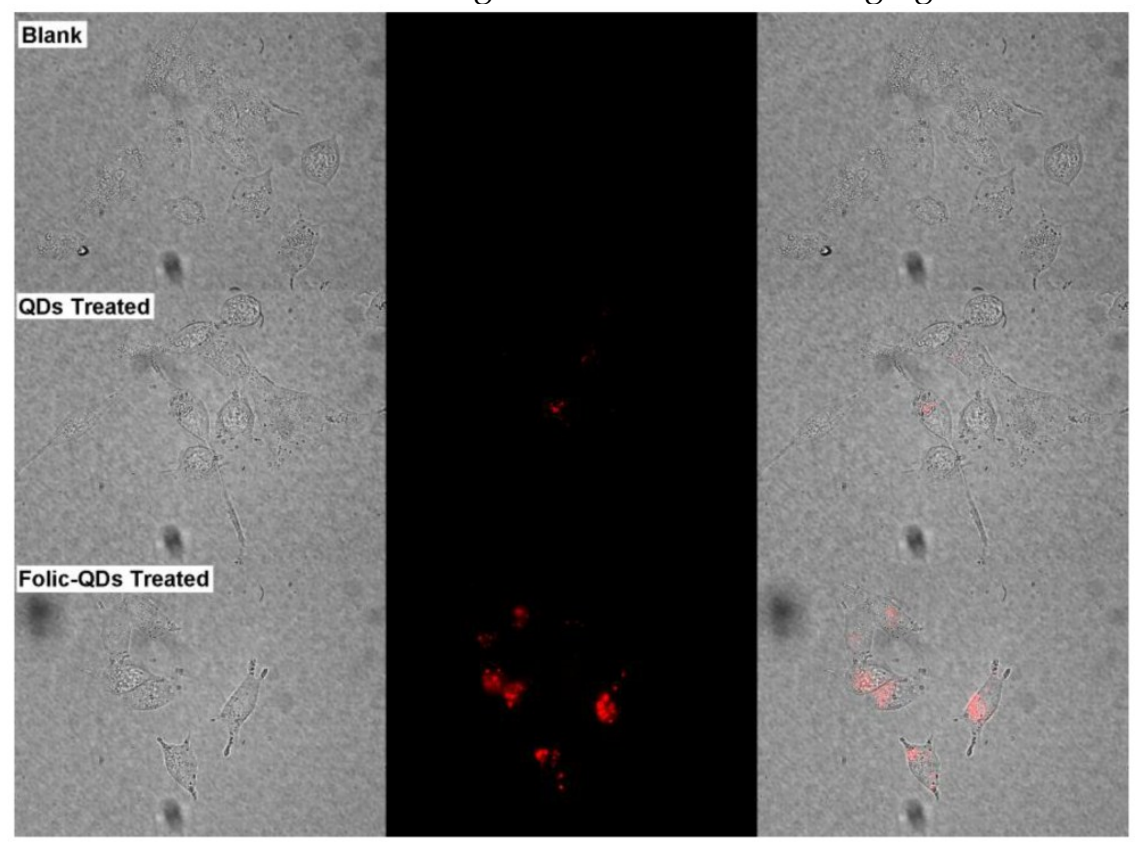

Figure 4. Imaging of live pancreatic cancer cells (Blank) as a comparison with which treated with folic acid conjugated phospholipid micelle encapsulated PbS QDs (Folic-QDs Treated) or non-conjugated QDs (QDs Treated). Left panel shows the bright-field image, middle panel is the fluorescent signal pseudo-colored in red and the right panel is the merged images.

Figure 5. Normalized characterization spectra from the QDs sample and the background signal of an untreated mouse, both under deep-red illumination. Inset in the upper left is the QDs sample in a centrifuge tube and the inset in the lower right shows the back of a mouse with its tail labeled. The two insets are pseudo-colored in red (QDs) and green (background).

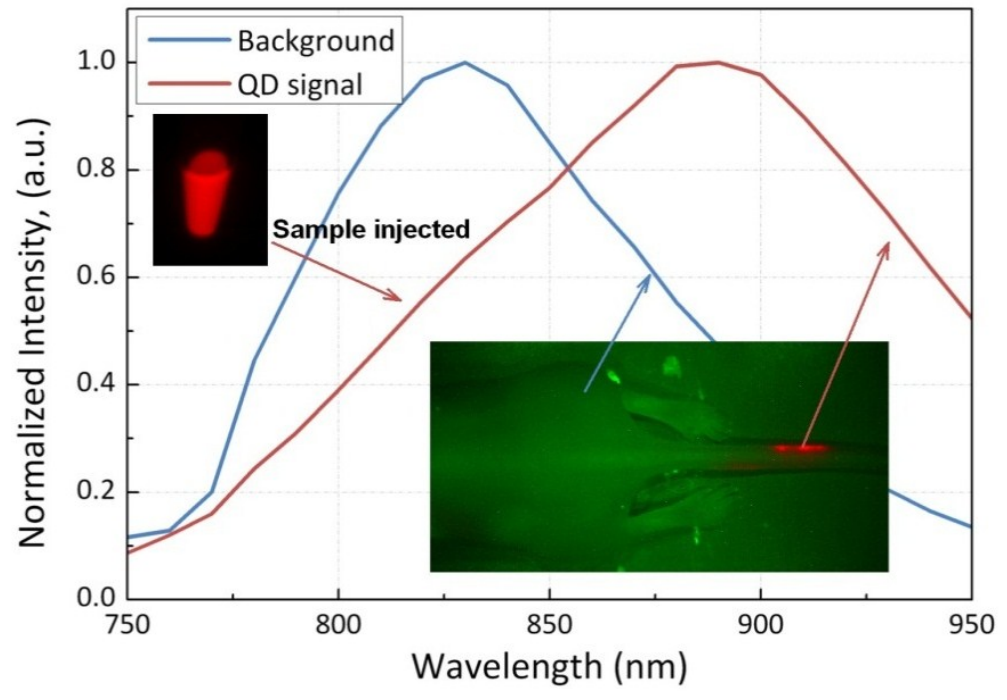




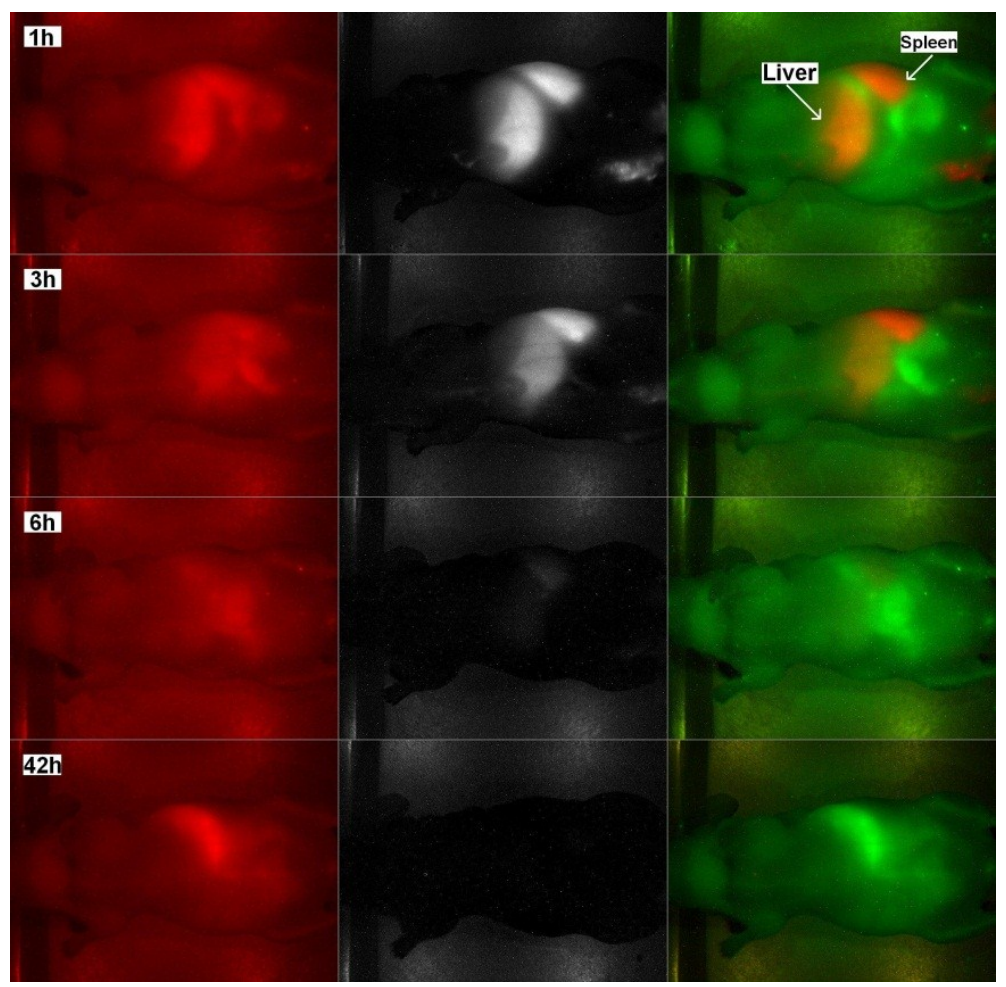

Figure 6. In vivo fluorescent images of a nude mouse tail vein injected with PEGylated phospholipid micelle encapsulated PbS QDs at Ih, $3 \mathrm{~h}, 6 \mathrm{~h}$ and $42 \mathrm{~h}$ after injection. Left panel shows the original unprocessed images, middle panel is the QDs signal channel and the panel to the right shows the pseudo-colored images of the spectrally separated signals, with background in green and the QDs signals in red.

The QDs toxicity has been widely discussed over the past few years especially for cadmium and lead-based QDs. In this work, lead-based QDs were used for in vitro and in vivo imaging. To examine the cytotoxicity of the QDs formulation, cell viability of Panc-1 cells was evaluated after treating them with QDs formulation. Figure 7 shows the results of the MTS assay of Panc-1 cells treated with the micelle-encapsulated PbS QDs. The cell viability of the Panc- 1 cells was maintained greater than $80 \%$ at particle concentration as high as $250 \mu \mathrm{g} / \mathrm{ml}$, for both $24 \mathrm{~h}$ and $48 \mathrm{~h}$ of post-treatment. This study reveals that the micelle-encapsulated PbS QDs is having a much lower cytotoxicity in comparison with polyanhydride coated PbSe QDs formulation [43], indicating that phospholipid micelle encapsulation is a useful strategy for producing low toxicity heavy-metal based QDs for some specific in vitro and in vivo applications.

We have also performed short term in vivo toxicity evaluation of the PEGylated phospholipid encapsulated PbS QDs using Balb/C mice. In this study, mice were administered with the QDs sample through tail vein injection at a concentration of $25 \mathrm{mg} / \mathrm{kg}$. Within 4 weeks of evaluation, we did not observe any changes in the drinking and eating be- havior of the mice. To further investigate the toxicity of the QDs formulation, the mice were sacrificed at 4 weeks after the injection and subsequently histological analysis was performed on the tissue samples of the major organs such as heart, liver, spleen, lung and kidney. These tissue section samples were processed and stained with hematoxylin and eosin (H\&E). As shown in Figure 8, no significant pathological changes were observed upon comparing the samples from treated mice to the control ones, demonstrating that the PEGylated phospholipid micelle-encapsulated $\mathrm{PbS}$ QDs did not cause any acute toxicity to the mice at a dosage of $25 \mathrm{mg} / \mathrm{kg}$.

The phospholipids micelle encapsulated QDs formulation offers an excellent platform for co-loading hydrophobic drugs in the micelle core for theranostic applications. To demonstrate the proof of concept of this idea, we encapsulated both PbS QDs and doxorubicin (Dox) using PEGylated phospholipids micelle. Figure 9 shows the accumulative release profile of Dox molecules from the micelle-encapsulated QDs/Dox formulation in comparison to phospholipid micelle encapsulated Dox only. The release profile was monitored for over 10 days. It was observed that both formulations show sustained release of Dox over the 10 days period. Interestingly, 
the release of Dox from the micelle-encapsulated QDs/Dox formulation has a higher release rate than that of the micelle-encapsulated Dox formulation during the first several hours and this is probably due to the faster diffusion rate created for Dox molecules in the presence of PbS QDs in the micelle core. To examine the therapeutic effect of the QDs/Dox formulation, in vitro cell viability studies were performed. Three formulations were examined in this study, namely, micelle-encapsulated QDs/Dox formulation, micelle-encapsulated Dox formulation and micelle-encapsulated QDs formulation. As

Figure 7. Relative cell viability of cells treated with PEGylated phospholipid micelle encapsulated PbS QDs of different concentrations, after $24 \mathrm{~h}$ and $48 \mathrm{~h}$. shown in figure 10, a significant decrease in the cell viability was observed for the Panc- 1 cells treated with either micelle-encapsulated QDs/Dox formulation or the micelle-encapsulated Dox formulation. The cell viability of the Panc- 1 cells was maintained greater than $90 \%$ for micelle-encapsulated QDs formulation. This experiment has highlighted the potential of using the micelle-encapsulated QDs/Dox formulation as theranostic probes for traceable targeted delivery and therapy applications.

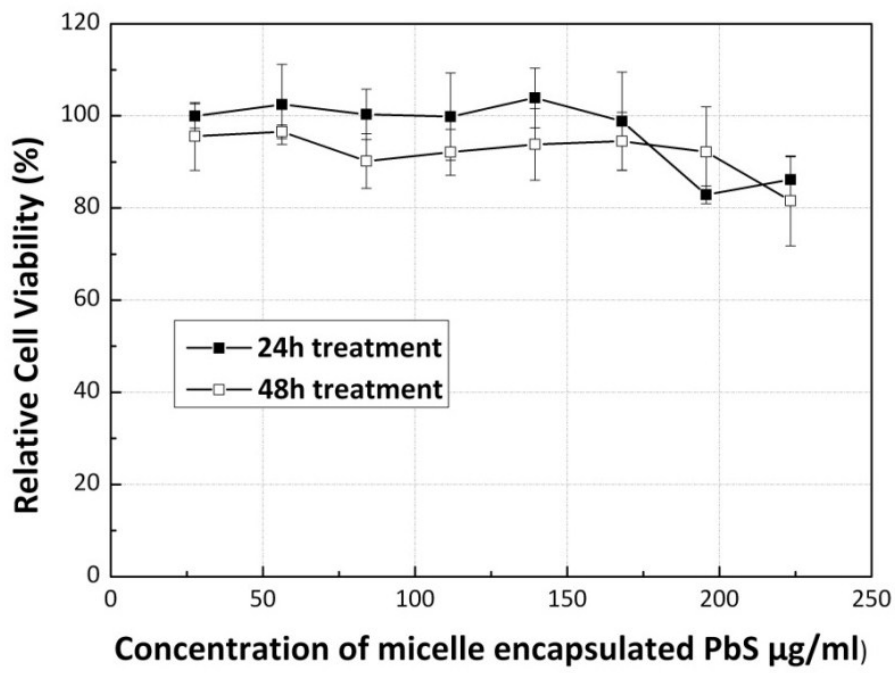

Figure 8. Histological studies on the major organs of the QDs injected mice at a dosage of $25 \mathrm{mg} / \mathrm{kg}$ after 4 weeks. No abnormal pathological changes were observable.

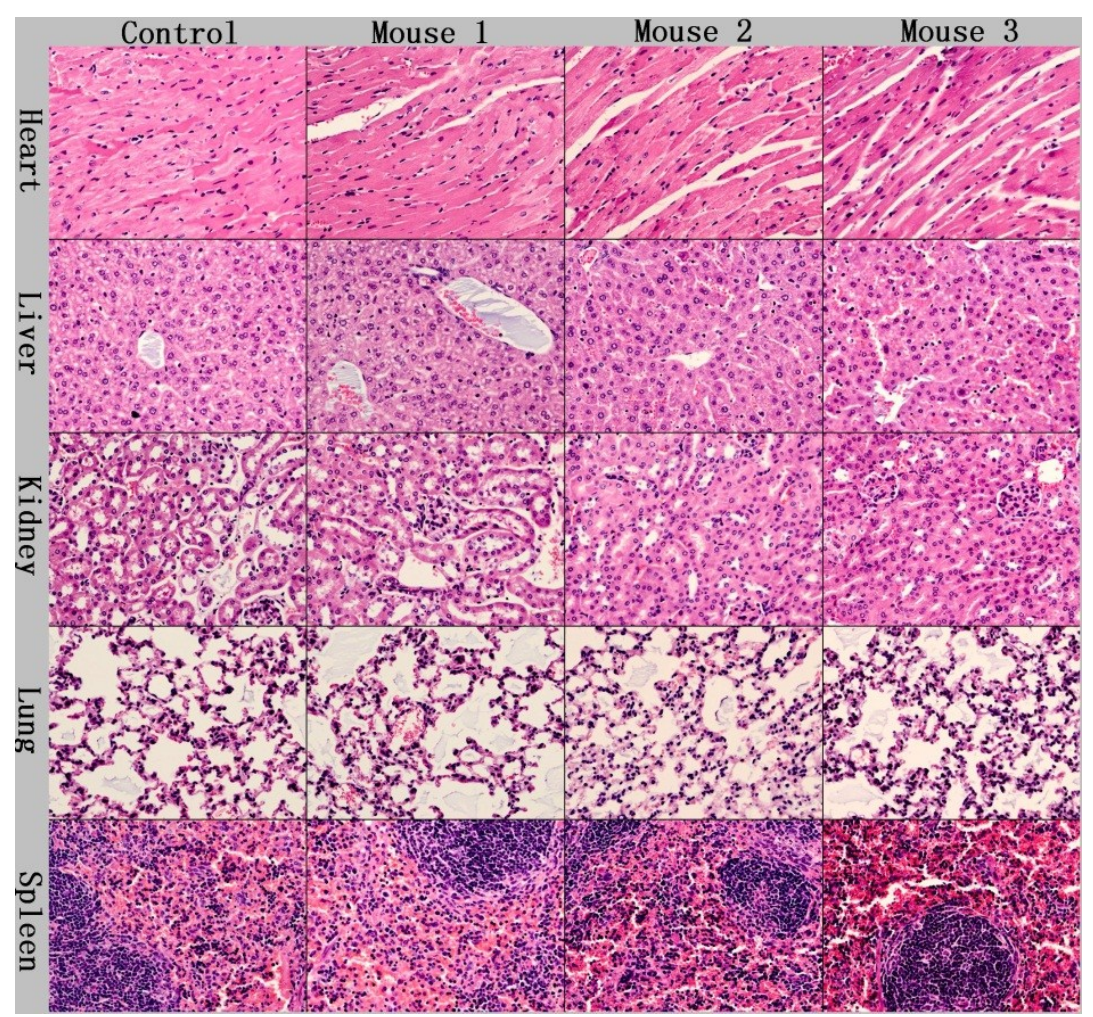




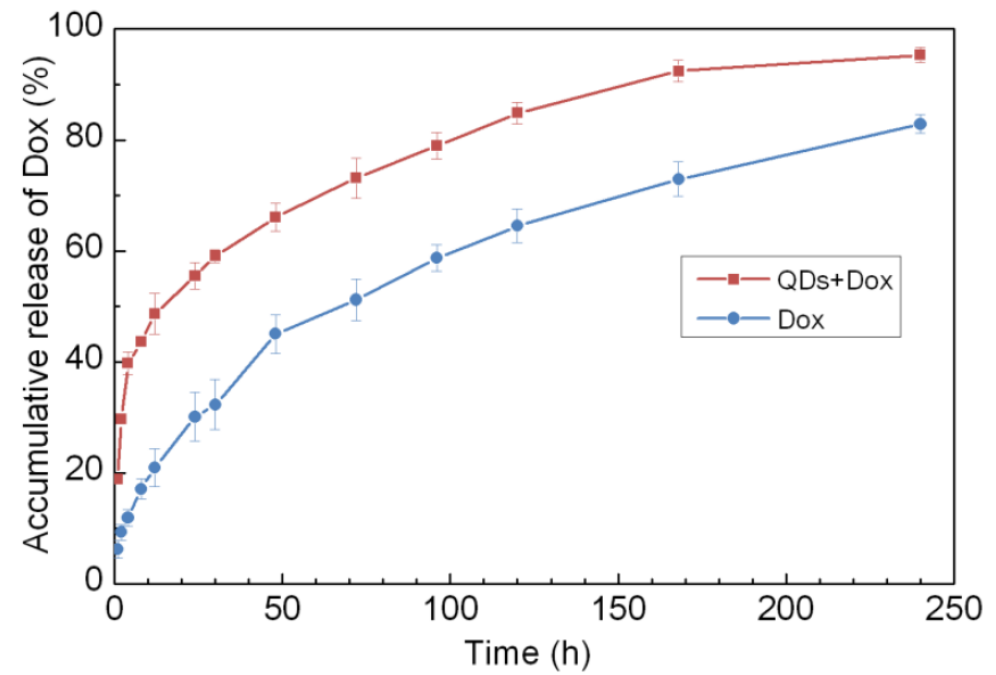

Figure 9. Accumulative release of Doxorubicin (Dox) from phospholipid micelle encapsulated formulations, with and without PbS QDs co-encapsulation.

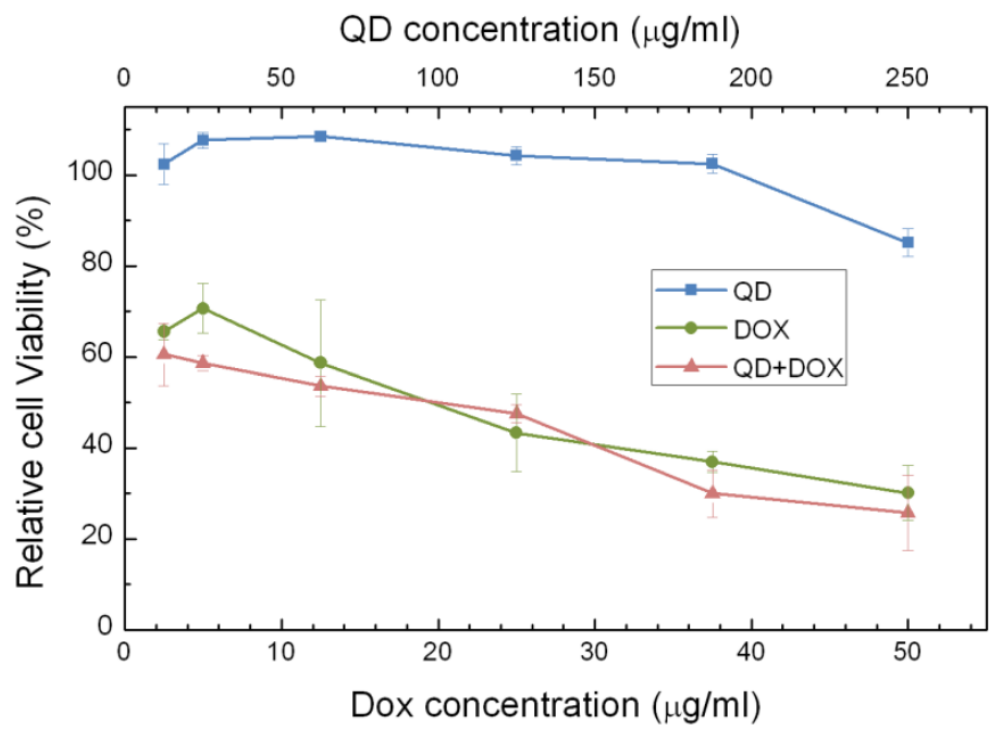

Figure 10. Relative cell viabilities of Panc-I celles treated with different formulations of phospholipid micelles, i.e., QDs encapsulated, Dox only encapsulated and QDs-Dox co-encapsulated, as a function of drug/QDs concentration $72 \mathrm{~h}$ following the treatment.

Heavy metals-based QDs, such as PbS, have not been widely used for biomedical applications due to their potential toxicity concern in biological systems. The result in this study has revealed that the toxicity of the PbS QDs can be significantly reduced by encapsulating them with phospholipid micelles and they can be served as an excellent tool for NIR imaging of cells and small animals. In addition, PbS QDs can be used as a good model to study the excretion of the functionalized nanoparticles in small animals through renal filtration since their size can be made smaller than $5 \mathrm{~nm}[44,45]$. Also, different coatings can be applied to the PbS QDs surface to investigate their excretion rate in vivo. This information will serve as guidelines for future development of excretable QDs formulation in vivo. More importantly, the NIR PbS QDs can be further functionalized with targeting ligands for NIR tumor imaging in small animals. Even though there are many advantages of using PbS QDs for basic biological research, we do not foresee that PbS QDs can be translated for biomedical and clinical applications since they are made from lead material which is considered to be harmful if the formulation breakdown in biological systems. Nonetheless, PbS QDs can be carefully tailored for some specific applications such as cell labeling, small animal imaging as 
well as flow cytometry study where toxicity concern will not be a major issue rather we are using the $\mathrm{PbS}$ QDs as a model probe to investigate some fundamental biological issues that only rely on cells and small animals model.

\section{Conclusion}

In summary, we have demonstrated that the PEGylated phospholipid micelle provided an effective nano-platform for encapsulating organic PbS QDs as contrast agents in NIR fluorescent imaging. The NIR fluorescent nanoparticles were successfully used for sensitive in vitro cell imaging as well as in vivo live animal imaging. To further address the toxicity issue introduced by the heavy metal containing QDs, we evaluated the cytotoxicity of the nanoparticles in cancer cells and the short term toxicity effect in small animals. The results reported here show that the micelle encapsulation has greatly reduced the toxicity of the PbS QDs, indicating that the encapsulation provides effective protection over the organic substances and the formulation used in our work can serve as a powerful nano-platform in the theranostics research.

\section{Acknowledgements}

This work was supported by the Start-up grant (M4080141.040) from Nanyang Technological University, and Tier 1 Academic Research Funds (M4010360.040 RG29/10) from Singapore Ministry of Education and partially from the Singapore Ministry of Ed ucation under Tier 2 Research Grant MOE2010-T2-2-010 (M4020020.040 ARC2/11).

\section{Competing Interests}

The authors have declared that no competing interest exists.

\section{References}

1. Zachariasse KA, Vanphuc N, Kozankiewicz B. Investigation of Micelles, Micro-Emulsions, and Phospholipid-Bilayers With the Pyridinium N-Phenolbetaine Et(30), A Polarity Probe for Aqueous Interfaces. J Phys Chem. 1981; 85: 2676-83. doi:10.1021/j150618a022.

2. Wendoloski JJ, Kimatian SJ, Schutt CE, Salemme FR. Molecular-Dynamics Simulation of a Phospholipid Micelle. Science. 1989; 243: 636-8. doi:10.1126/science.2916118.

3. Bangham AD, Horne RW. Negative staining of phospholipids and their structural modification by surface-active agents as observed in the electron microscope. Journal of Molecular Biology. 1964; 8: 660-IN10. doi:10.1016/s0022-2836(64)80115-7.

4. Gabizon A, Shmeeda H, Horowitz AT, Zalipsky S. Tumor cell targeting of liposome-entrapped drugs with phospholipid-anchored folic acid-PEG conjugates. Adv Drug Deliv Rev. 2004; 56: 1177-92. doi:10.1016/j.addr.2004.01.011.

5. Walsh TJ, Finberg RW, Arndt C, Hiemenz J, Schwartz C, Bodensteiner D, et al. Liposomal Amphotericin B for Empirical Therapy in Patients with Persistent Fever and Neutropenia. New England Journal of Medicine. 1999; 340: 764-71. doi:doi:10.1056/NEJM199903113401004.

6. Maherani B, Arab-Tehrany E, Linder M. Mechanism of Bioactive Transfer through Liposomal Bilayers. Curr Drug Targets. 2011; 12: 531-45. doi:10.2174/138945011794751582.
7. Fricker G, Kromp T, Wendel A, Blume A, Zirkel J, Rebmann H, et al. Phospholipids and Lipid-Based Formulations in Oral Drug Delivery. Pharm Res. 2010; 27: 1469-86. doi:10.1007/s11095-010-0130-x.

8. Kwon GS. Polymeric micelles for delivery of poorly water-soluble compounds. Crit Rev Ther Drug Carr Syst. 2003; 20: 357-403. doi:10.1615/CritRevTherDrugCarrierSyst.v20.i5.20.

9. Krishnadas A, Rubinstein I, Onyuksel H. Sterically stabilized phospholipid mixed micelles: In vitro evaluation as a novel carrier for water-insoluble drugs. Pharm Res. 2003; 20: 297-302. doi:10.1023/a:1022243709003.

10. Ashok B, Arleth L, Hjelm RP, Rubinstein I, Onyuksel H. In vitro characterization of PEGylated phospholipid micelles for improved drug solubilization: Effects of PEG chain length and PC incorporation. J Pharm Sci. 2004; 93: 2476-87. doi:10.1002/jps.20150.

11. Lukyanov AN, Gao ZG, Mazzola L, Torchilin VP. Polyethylene glycol-diacyllipid micelles demonstrate increased acculumation in subcutaneous tumors in mice. Pharm Res. 2002; 19: 1424-9. doi:10.1023/a:1020488012264.

12. Prencipe G, Tabakman SM, Welsher K, Liu Z, Goodwin AP, Zhang L, et al. PEG Branched Polymer for Functionalization of Nanomaterials with Ultralong Blood Circulation. J Am Chem Soc. 2009; 131: 4783-7. doi:10.1021/ja809086q.

13. Ghoroghchian PP, Frail PR, Susumu K, Blessington D, Brannan AK, Bates FS, et al. Near-infrared-emissive polymersomes: Self-assembled soft matter for in vivo optical imaging. Proceedings of the National Academy of Sciences of the United States of America. 2005; 102: 2922-7. doi:10.1073/pnas.0409394102.

14. Licha $\mathrm{K}$, Olbrich $\mathrm{C}$. Optical imaging in drug discovery and diagnostic applications. Adv Drug Deliv Rev. 2005; 57: 1087-108. doi:10.1016/j.addr.2005.01.021.

15. Kim J, Piao Y, Hyeon T. Multifunctional nanostructured materials for multimodal imaging, and simultaneous imaging and therapy. Chemical Society Reviews. 2009; 38: 372-90. doi:10.1039/b709883a.

16. Tsien RY. The green fluorescent protein. Annual Review of Biochemistry. 1998; 67: 509-44. doi:10.1146/annurev.biochem.67.1.509.

17. Shaner NC, Campbell RE, Steinbach PA, Giepmans BNG, Palmer AE, Tsien RY. Improved monomeric red, orange and yellow fluorescent proteins derived from Discosoma sp red fluorescent protein. Nature Biotechnology. 2004; 22: 1567-72. doi:10.1038/nbt1037.

18. Rao JH, Dragulescu-Andrasi A, Yao HQ. Fluorescence imaging in vivo: recent advances. Current Opinion in Biotechnology. 2007; 18: 17-25. doi:10.1016/j.copbio.2007.01.003.

19. Prasad PN. Bioimaging: Principles and Techniques; Introduction to Biophotonics. John Wiley \& Sons, Inc. 2004: 203-54.

20. Ntziachristos V, Bremer C, Weissleder R. Fluorescence imaging with near-infrared light: new technological advances that enable in vivo molecular imaging. European Radiology. 2003; 13: 195-208. doi:10.1007/s00330-002-1524-x.

21. Sevick-Muraca EM, Houston JP, Gurfinkel M. Fluorescence-enhanced, near infrared diagnostic imaging with contrast agents. Current Opinion in Chemical Biology. 2002; 6: 642-50. doi:10.1016/s1367-5931(02)00356-3.

22. Tung $\mathrm{CH}$, Mahmood $\mathrm{U}$, Bredow $\mathrm{S}$, Weissleder R. In vivo imaging of proteolytic enzyme activity using a novel molecular reporter. Cancer Research. 2000; 60: 4953-8.

23. Zaheer A, Lenkinski RE, Mahmood A, Jones AG, Cantley LC, Frangioni JV. In vivo near-infrared fluorescence imaging of osteoblastic activity. Nature Biotechnology. 2001; 19: 1148-54. doi:10.1038/nbt1201-1148.

24. Weissleder R. A clearer vision for in vivo imaging. Nat Biotech. 2001; 19 : 316-7.

25. Cai W, Shin D-W, Chen K, Gheysens O, Cao Q, Wang SX, et al. Peptide-Labeled Near-Infrared Quantum Dots for Imaging Tumor Vasculature in Living Subjects. Nano Letters. 2006; 6: 669-76. doi:10.1021/nl052405t.

26. Michalet X, Pinaud FF, Bentolila LA, Tsay JM, Doose S, Li JJ, et al. Quantum Dots for Live Cells, in Vivo Imaging, and Diagnostics. Science. 2005; 307: 538-44. doi:10.1126/science.1104274.

27. Prasad PN. Quantum-Confined Materials; Nanophotonics. John Wiley \& Sons, Inc. 2004:79-127.

28. Dubertret B, Skourides P, Norris DJ, Noireaux V, Brivanlou AH, Libchaber A. In vivo imaging of quantum dots encapsulated in phospholipid micelles. Science. 2002; 298: 1759-62. doi:10.1126/science.1077194.

29. Bruchez M, Moronne M, Gin P, Weiss S, Alivisatos AP. Semiconductor nanocrystals as fluorescent biological labels. Science. 1998; 281: 2013-6. doi:10.1126/science.281.5385.2013. 
30. Nirmal M, Dabbousi BO, Bawendi MG, Macklin JJ, Trautman JK, Harris $\mathrm{TD}$, et al. Fluorescence intermittency in single cadmium selenide nanocrystals. Nature. 1996; 383: 802-4. doi:10.1038/383802a0.

31. Rabani E, Hetenyi B, Berne BJ, Brus LE. Electronic properties of CdSe nanocrystals in the absence and presence of a dielectric medium. Journal of Chemical Physics. 1999; 110: 5355-69. doi:10.1063/1.478431.

32. Alivisatos AP. Semiconductor clusters, nanocrystals, and quantum dots. Science. 1996; 271: 933-7. doi:10.1126/science.271.5251.933.

33. Murray CB, Kagan CR, Bawendi MG. Synthesis and characterization of monodisperse nanocrystals and close-packed nanocrystal assemblies. Annual Review of Materials Science. 2000; 30: 545-610. doi:10.1146/annurev.matsci.30.1.545.

34. Medintz IL, Uyeda HT, Goldman ER, Mattoussi H. Quantum dot bioconjugates for imaging, labelling and sensing. Nature Materials. 2005; 4: 435-46. doi:10.1038/nmat1390.

35. Lee J, Ji K, Kim J, Park C, Lim KH, Yoon TH, et al. Acute toxicity of two $\mathrm{CdSe} / \mathrm{ZnSe}$ quantum dots with different surface coating in Daphnia magna under various light conditions. Environmental Toxicology. 2010; 25: 593-600. doi:10.1002/tox.20520.

36. Lewinski N, Colvin V, Drezek R. Cytotoxicity of Nanoparticles. Small. 2008; 4: 26-49. doi:10.1002/smll.200700595.

37. Yong K-T, Roy I, Ding H, Bergey EJ, Prasad PN. Biocompatible Near-Infrared Quantum Dots as Ultrasensitive Probes for Long-Term in vivo Imaging Applications. Small. 2009; 5: 1997-2004. doi:10.1002/smll.200900547.

38. Truong L, Moody I, Stankus D, Nason J, Lonergan M, Tanguay R. Differential stability of lead sulfide nanoparticles influences biological responses in embryonic zebrafish. Archives of Toxicology. 2011; 85: 787-98. doi:10.1007/s00204-010-0627-4.

39. Cory AH, Owen TC, Barltrop JA, Cory JG. Use of an Aqueous Soluble Tetrazolium Formazan Assay for Cell-Growth Assays in Culture. Cancer Communications. 1991; 3: 207-12.

40. Buttke TM, McCubrey JA, Owen TC. Use of an Aqueous Soluble Tetrazolium Formazan Assay to Measure Viability and Proliferation of Lymphokine-Dependent Cell-Lines. J Immunol Methods. 1993; 157: 233-40. doi:10.1016/0022-1759(93)90092-1.

41. Lee RJ, Low PS. FOLATE-MEDIATED TUMOR-CELL TARGETING OF LIPOSOME-ENTRAPPED DOXORUBICIN IN-VITRO. Biochimica Et Biophysica Acta-Biomembranes. 1995; 1233: 134-44. doi:10.1016/0005-2736(94)00235-h.

42. Kim YI. Folate and carcinogenesis: Evidence, mechanisms, and implications. Journal of Nutritional Biochemistry. 1999; 10: 66-88. doi:10.1016/s0955-2863(98)00074-6.

43. Tan TT, Selvan ST, Zhao L, Gao S, Ying JY. Size Control, Shape Evolution, and Silica Coating of Near-Infrared-Emitting PbSe Quantum Dots. Chemistry of Materials. 2007; 19: 3112-7. doi:10.1021/cm061974e.

44. Choi HS, Ipe BI, Misra P, Lee JH, Bawendi MG, Frangioni JV. Tissue- and Organ-Selective Biodistribution of NIR Fluorescent Quantum Dots. Nano Letters. 2009; 9: 2354-9. doi:10.1021/n1900872r.

45. Choi HS, Liu W, Misra P, Tanaka E, Zimmer JP, Ipe BI, et al. Renal clearance of quantum dots. Nature Biotechnology. 2007; 25: 1165-70. doi:10.1038/nbt1340. 